\title{
In-Flight CCD Distortion Calibration for Pushbroom Satellites Based on Subpixel Correlation
}

\author{
Sébastien Leprince, Student Member, IEEE, Pablo Musé, and Jean-Philippe Avouac
}

\begin{abstract}
We describe a method that allows for accurate inflight calibration of the interior orientation of any pushbroom camera and that in particular solves the problem of modeling the distortions induced by charge coupled device (CCD) misalignments. The distortion induced on the ground by each $\mathrm{CCD}$ is measured using subpixel correlation between the orthorectified image to be calibrated and an orthorectified reference image that is assumed distortion free. Distortions are modeled as camera defects, which are assumed constant over time. Our results show that in-flight interior orientation calibration reduces internal camera biases by one order of magnitude. In particular, we fully characterize and model the Satellite Pour l'Observation de la Terre (SPOT) 4-HRV1 sensor, and we conjecture that distortions mostly result from the mechanical strain produced when the satellite was launched rather than from effects of on-orbit thermal variations or aging. The derived calibration models have been integrated to the software package Coregistration of Optically Sensed Images and Correlation (COSI-Corr), freely available from the Caltech Tectonics Observatory website. Such calibration models are particularly useful in reducing biases in digital elevation models (DEMs) generated from stereo matching and in improving the accuracy of change detection algorithms.
\end{abstract}

Index Terms-Change detection, charge coupled device (CCD) distortions, ground deformations, interior orientation, orthorectification, pushbroom camera calibration, Satellite Pour l'Observation de la Terre (SPOT), subpixel correlation.

\section{INTRODUCTION}

$\mathbf{R}$ ECENT methodological advances have made it possible to accurately orthorectify and coregister pairs of optical satellite images, acquired from pushbroom systems, on an operational basis [1], [2]. The average coregistration accuracy is on the order of $1 / 50$ of the pixel size, and associated with an accurate subpixel correlation technique, the quantitative monitoring of the Earth's surface deformations has become possible. For instance, the Coregistration of Optically Sensed Images and Correlation (COSI-Corr) (http://www. tectonics.caltech.edu/slip_history/spot_coseis) technique has been successfully applied to measure the horizontal coseismic displacement field induced by large earthquakes [1]-[7], glacier flow [8]-[10], landslides [8], and sand dune migrations [11]. In each case, the accuracy on the horizontal displacement measured was on the order of $1 / 10$ of the pixel size for individual

Manuscript received October 22, 2007; revised January 15, 2008. This work was supported in part by the National Science Foundation under Grant EAR0409652 and Grant EAR-0636097, and in part by the Gordon and Betty Moore Foundation.

S. Leprince is with the Department of Electrical Engineering, California Institute of Technology, Pasadena, CA 91125 USA (e-mail: leprincs@ caltech.edu).

P. Musé and J.-P. Avouac are with the Division of Geological and Planetary Sciences, California Institute of Technology, Pasadena, CA 91125 USA.

Digital Object Identifier 10.1109/TGRS.2008.918649 measurements and often better than $1 / 20$ of the pixel size for measurements stacked over a swath.

At this level of accuracy, the ground deformation measurements are sensitive to any potential defect in the physical modeling of the satellite system. One recurring and troublesome issue has been the proper modeling of the internal orientation of pushbroom imaging satellites, whose detector array is not composed of a single charge coupled device (CCD) line array but rather of several line arrays combined together to form a longer single one. This is, for instance, the case of the Satellite Pour l'Observation de la Terre (SPOT) satellites 1, 2, 3, and 4, where the panchromatic bands are acquired using four CCD line arrays of 1500 pixels each, combined together through an optical divider to form the complete equivalent 6000-pixel line scanning array [12].

Artifacts due to the improper modeling of CCD's alignment are shown in Fig. 1. This figure shows the displacement field measured from the subpixel correlation of a SPOT 4-HRV1 panchromatic image acquired in 1998 and a SPOT 2-HRV1 panchromatic image acquired in 2000, which were first precisely coregistered and orthorectified. These images cover the epicentral area of the Mw 7.1, Hector Mine earthquake, California, which struck in 1999, rupturing the Earth's surface over a distance of about $60 \mathrm{~km}$. Fig. 1 should then represent the measurement of the horizontal coseismic displacement field induced by this earthquake. The main rupture is indeed revealed and can be mapped in detail, but measurements seem biased by linear artifacts running in the satellites' along-track direction. In areas where the coseismic deformation is negligible, biases corresponding to up to $1.6 \mathrm{~m}$ of ground displacement are measured. They are caused by the contribution of misaligned and distorted CCD line arrays of both satellites that were not properly modeled during orthorectification. A distortion-free perfectly straight CCD line array model was indeed used.

In this paper, we describe a method that allows for in-flight calibration of the interior orientation of any pushbroom camera and that in particular solves the problem of modeling the distortions induced by individual CCD misalignments. Our discussion and results are illustrated using pairs of panchromatic SPOT images. Section II presents a review of how the CCD distortion problem has been addressed thus far. In Section III, we expose our calibration methodology, which is based on the measurement of the CCD-induced ground distortions using subpixel correlation and on interpreting them as errors on the camera model. Internal orientation distortions are assumed constant over time and are measured with respect to an image that is assumed distortion free. Section IV presents the results obtained, whereas Section V discusses the performances and limitations of our calibration method. 


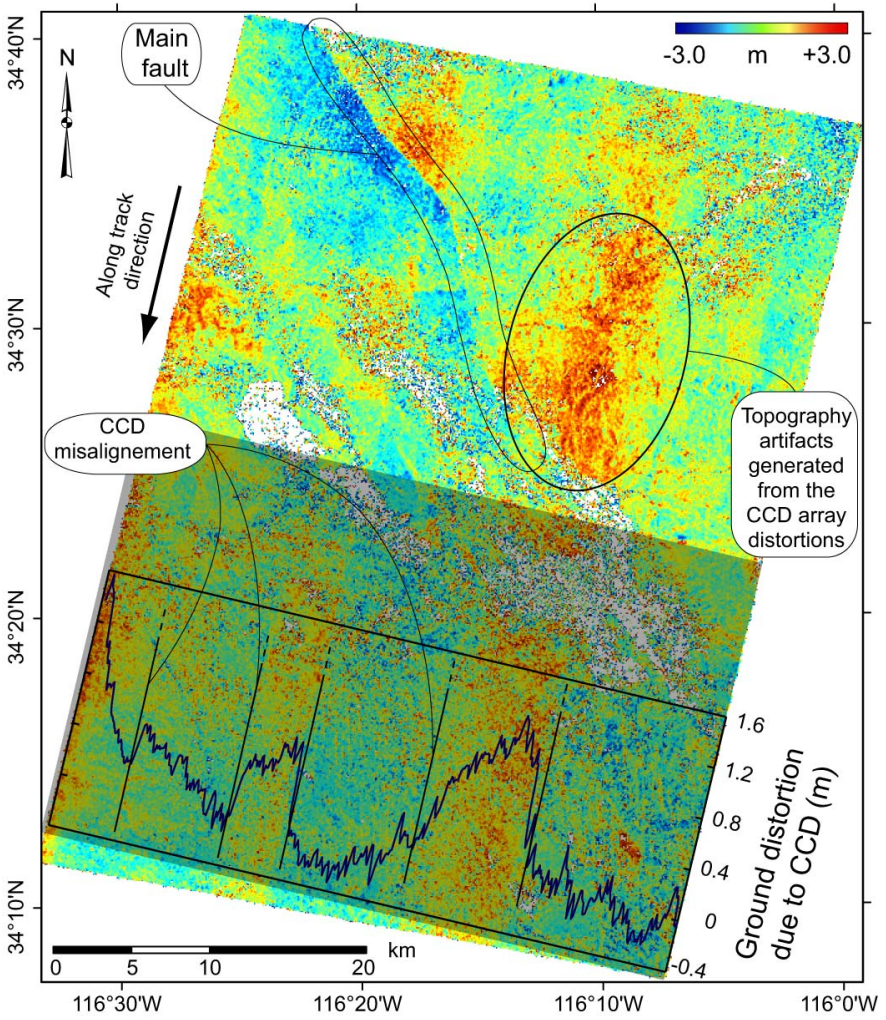

Fig. 1. East-west component (eastward positive) of the displacement field measured over the Hector Mine area, California, using a 10-m panchromatic SPOT 4 image acquired on August 17, 1998, and a 10-m panchromatic SPOT 2 image acquired on August 10, 2009. The images were coregistered with the topography using the $1 / 3$ arcsec $\sim 9$ m NED DEM from USGS, coregistered together using automatic subpixel GCP selection, and orthorectified on a $10-\mathrm{m}$ UTM grid. Subpixel correlation was performed using $32 \times 32$ pixel correlation windows, sliding with a step of eight pixels, yielding a displacement map sampled at every $80 \mathrm{~m}$. This experiment is the same as the one depicted in [1, Fig. 24]. The fault rupture, induced by the Mw 7.1, 1999 Hector Mine earthquake, is characterized as a discontinuity in the displacement field and accounts for up to $5.5 \mathrm{~m}$ of surface offset in this component. No measurement is assigned to white points, where correlation was lost due to drastic changes over alluvial areas, or because of sensor saturation on white sandy areas. Linear artifacts, running in the satellites' along-track direction, betray the unmodeled distortions of the CCD arrays of each satellite. The dark box represents an area where the tectonic signal is assumed negligible with respect to the measurement noise $(\sim 70 \mathrm{~cm})$. The superimposed graph shows the displacements within this box, averaged in the along-track direction. This stacked profile estimates the bias induced by the CCD distortions of both satellites. In flat topography areas (this is mostly the case in the dark box), the induced ground distortions are up to $1.6 \mathrm{~m}$. However, these also depend on the topography variations, as seen in the circled area, and as explained in Fig. 2.

\section{Previous WORK}

To our knowledge, there exists very few authors in the open literature who have explicitly documented the problem of correcting the CCD distortions of pushbroom satellites. They all based their studies on the SPOT satellites and are described below.

\section{A. Benefiting From the Dual Acquisition System HRV1 and HRV2 on Spot Satellites}

In [13] and [14], Westin describes a procedure to explicitly calibrate the CCD distortions of the SPOT 1 satellite. The SPOT 1, 2, 3, and 4 satellites are equipped with two similar instruments (HRV1 and HRV2) that can simultaneously acquire images and with different pointing angles thanks to their in- dependent steering mirrors. Such acquisitions constitute stereo pairs with a base-to-height ratio equal to 0 , and once projected on a common reference system [universal transverse mercator (UTM)], the local disparities are formulated as the superposition of the distortions from both instruments. Distortions from each instrument can be separated using at least three such pairs, each pair having a different incidence angle difference. This technique works well but is of limited practical use. One practical problem is that the HRV1 and HRV2 instruments are usually not simultaneously acquiring scenes, and finding at least three such stereo pairs from the SPOT archives is challenging. However, this problem could easily be overcome by a special order of images if the satellite is still running. Another more serious limitation is that this method applies only to satellites having two optical systems that can simultaneously image the same area. In other words, this formulation is only valid for SPOT satellites, whereas other satellites such as Quickbird, Ikonos, the IRS-1C satellite, etc., which do not possess a duplicate of their optical instrument, but which also exhibit troublesome CCD distortions [15], [16], could benefit from a more general and versatile approach.

\section{B. Along-Track Subtraction of Stacked Profiles}

To correct the CCD-induced distortions in a correlation image like the one shown in Fig. 1, one may be tempted to simply use postprocessing tools to remove the apparent artifacts. The exact and tedious modeling of the distortions then becomes unnecessary. For instance, one could think of removing the CCD artifacts from Fig. 1 by subtracting, in the satellites' along-track direction, stacked profiles taken where the displacement field is assumed to be zero. This is equivalent to subtracting the graph superimposed in Fig. 1 from the whole correlation image. Unfortunately, this method, proposed in [4], has two major drawbacks. First, the correlation image must possess large areas where the ground displacement is negligible, which is impractical in the case of images spanning a large earthquake. Second, this stacking technique simply does not work because, as explained in Fig. 2, the CCD-induced distortions on the ground depend on the topography. Hence, averaging nonconstant ground distortions is meaningless. As an illustration, the circled artifacts in Fig. 1 cannot be canceled from stack subtraction since they show obvious heterogeneities in the along-track direction. These parallax artifacts result from the CCD distortions and cannot be the result of the digital elevation model (DEM) vertical inaccuracy. The pre and post earthquake images have an incidence angle difference of $8.1^{\circ}$ and the national elevation dataset (NED) DEM has a vertical accuracy within $\sim 3 \mathrm{~m}$ [17]. Consequently, the ground disparity induced by the DEM parallax should not exceed $42 \mathrm{~cm}$, and the ground disparities measured are comprised between 2 and $3 \mathrm{~m}$. Hence, the topography-dependent artifacts circled in Fig. 1 are indeed produced by the CCD distortions.

\section{Proposed Methodology}

\section{A. Assumptions and Notations}

In this paper, we assume that the artifacts observed in the correlation images are due to a combination of nonseparable 


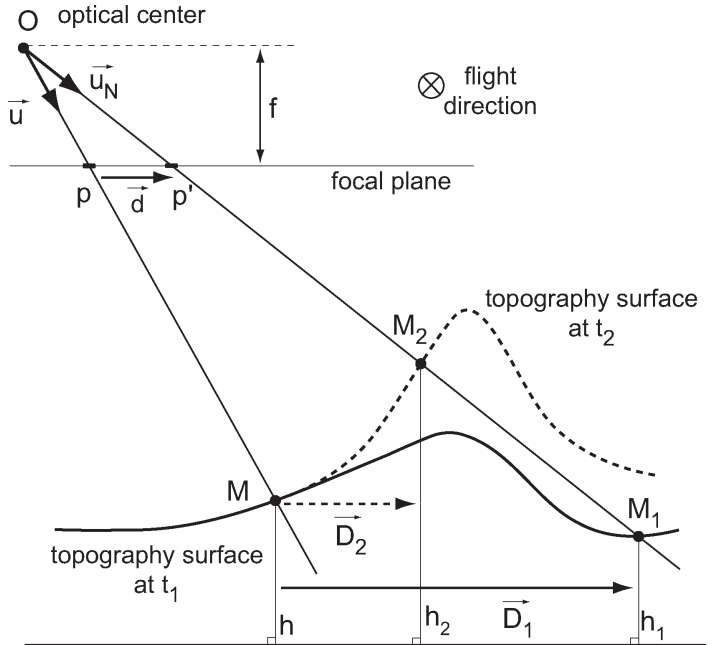

Earth projective reference

Fig. 2. Assume that a particular pixel is thought to be imaging a scene from the position $p$ in the satellite focal plane. In addition, assume that the optical system is sustaining some distortion, constant over time, that can be modeled as if this particular pixel $p$ was in fact seeing the scene from the position $p^{\prime}$ in the focal plane. Call this distortion $\vec{d}=p \vec{p}^{\prime}$. Then, orthorectify, coregister, and correlate the distorted image with a distortion-free reference image. If at a particular acquisition time $t_{1}$ the topography of the scene is represented by the solid black line, then the ground disparity measured from correlation is $\vec{D}_{1}$. Since $\vec{d}$ is assumed constant over time, if the topography is instead represented by the dotted line at time $t_{2}$, the ground disparity measured will be $\vec{D}_{2} \neq \vec{D}_{1}$. Hence, the ground disparities measured from the correlation of orthorectified images depend on the topography variations and cannot be averaged. This makes clear that CCD distortions must bias the production of DEMs from stereoscopic images [13]. The distortion in the focal plane $\vec{d}$ is retrieved using the observed ground distortion. If at time $t_{1}$ the pixel $p$ sees the ground point $M$ and the ground disparity $\vec{D}_{1}$ is measured, it means that the pixel $p$ should have seen the ground point $M_{1}=M+\vec{D}_{1}$ at elevation $h_{1}$ instead. The problem of determining $\vec{d}$ is therefore equivalent to determining the new camera unitary pointing vector $\vec{u}_{N}$ of the pixel $p$, such that $p$ sees the ground point $M_{1}$ when projected according to $\vec{u}_{N}$.

distortions from the optical system and the CCD elements, and they can all be modeled as positioning errors on the CCD element location in the focal plane [13]. For a given CCD element, the distortion is considered constant over the time of acquisition of an image. The acquisition time is about $9 \mathrm{~s}$ for a SPOT image. We will discuss the stationarity of CCD distortions over longer periods in Section V.

To express our internal orientation model that accounts for the CCD and optical distortions, we use the internal orientation representation developed for the SPOT satellites, where each CCD element is associated with a particular look direction [12]. The notations are reported in Fig. 3.

The calibrated interior orientation of a slave image is derived from the correlation analysis between the slave image and a reference image that is assumed bias free and perfectly orthorectified. This reference image should largely overlap with the slave image to be calibrated. For example, it could be a mosaic of high-resolution aerial photographs orthorectified at the resolution of the slave image [18]. In this paper, the reference image is a SPOT 5-HRG1 panchromatic image. The SPOT 5 sensor is composed of a single CCD line array that is accurately calibrated [18] and that has shown no detectable bias during correlation analysis [1]. The 1/3 $\operatorname{arcsec}(\sim 9 \mathrm{~m})$ NED

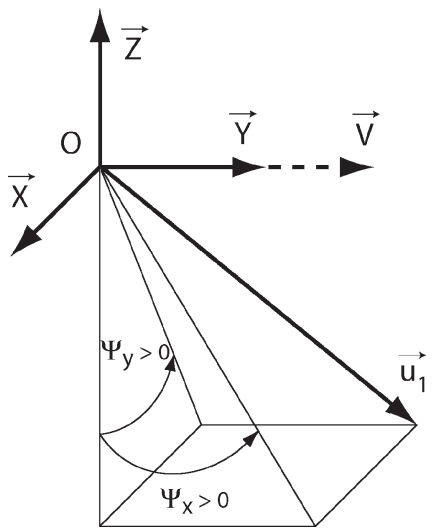

Fig. 3. Each CCD element $p$ in the focal plane is characterized by a pointing direction $\vec{u}_{1}$, with origin as the instrument's optical center $O$. These look directions are derived from the look angles $\left(\Psi_{x}, \Psi_{y}\right)$, such that $\vec{u}_{1}(p)=$ $\left[-\tan \psi_{y}(p), \tan \psi_{x}(p),-1\right]^{T} / K$, and with $K$ such that $\left\|\vec{u}_{1}(p)\right\|_{2}=1$, for all $p$. The set of look directions is fixed over a given acquisition and models the satellite interior orientation. It is given in the spacecraft body fixed reference system, also called the Navigation Reference Coordinate System. At nominal attitude, when the satellite roll, pitch, and yaw are null angles, we have $\vec{Y} / / \vec{V}$, $\vec{Z} / / \vec{P}$, and $\vec{X}=\vec{Y} \times \vec{Z}$, if $\vec{P}$ and $\vec{V}$ denote the satellite position and velocity vectors, respectively.

DEM [17] is used for orthorectification purpose. Its resolution is thought to be sufficient to produce orthorectified images with negligible parallax effects if the images are free of modeling distortions.

\section{B. Methodology}

CCD distortions induce subtle disparities in the images that account for at most a small fraction of the pixel size. Therefore, the slave raw image should be perfectly orthorectified and coregistered with the reference, except for the CCD distortions that should be unambiguously measured. This is achieved thanks to accurate ground control points (GCPs) that correct for the satellite exterior orientation errors and that are generated between the raw slave image and the orthorectified reference using subpixel correlation [1].

The result of the correlation analysis between a SPOT 5-HRG1 panchromatic reference image and a SPOT 4-HRV1 slave image is shown in Fig. 4. The disparity field shows linear artifacts attributed to the SPOT 4 CCD distortions. No other biases are visible, meaning that precise orthorectification and coregistration have been achieved. In particular, the exterior orientation is satisfyingly modeled as no oscillating pattern is observed (typical for roll, pitch, or yaw variation residuals [2], [19]).

1) Orthorectification Model: The orthorectification model, as detailed in [1], is of the form

$$
M(p)=O(p)+\lambda\left[T(p) R(p) \vec{u}_{1}(p)+\vec{\delta}(p)\right]
$$

where

$M \quad$ point on the ground seen by the pixel $p$;

$O$ position of the optical center in space when $p$ was being acquired;

$\vec{u}_{1} \quad$ interior look direction of the pixel $p$ as defined in Fig. 3; 


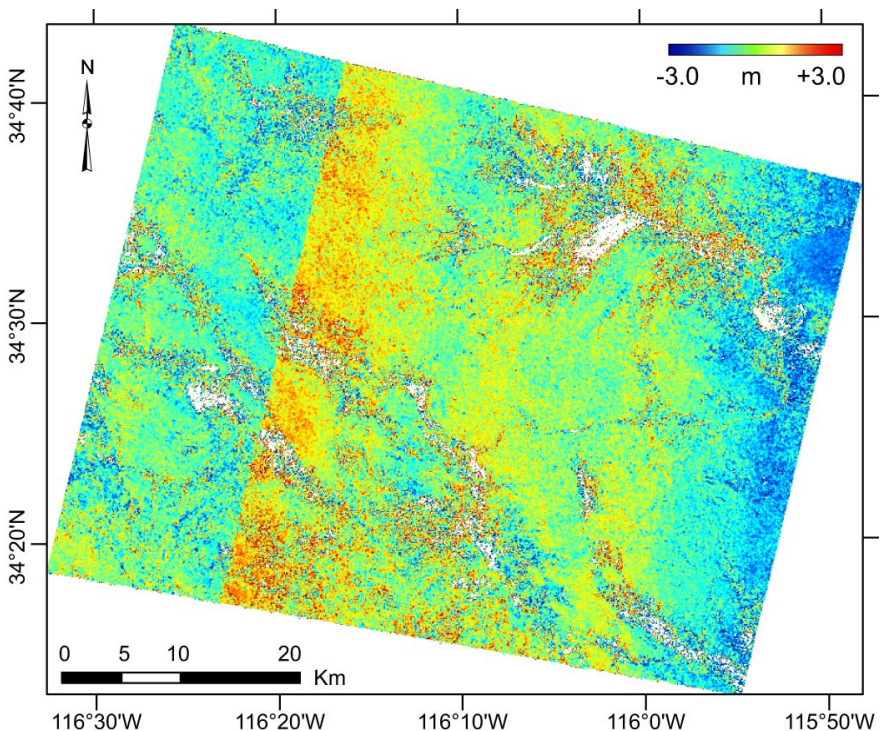

Fig. 4. East-west component (eastward positive) of the disparity field measured from the subpixel correlation of a reference SPOT 5-HRG1 5-m panchromatic image acquired on January 24, 2003 and a slave SPOT 4-HRV1 10-m panchromatic image acquired on March 11, 2000. Both images were orthorectified using the 1/3 arcsec NED DEM from USGS. Thirty subpixel GCPs were used to tie the SPOT 5 image to the DEM, and six subpixel GCPs were used to tie both orthorectified images together [1]. Correlation analysis was performed on $32 \times 32$ pixel windows, sliding with a step of 8 pixels $(80 \mathrm{~m}$ on the ground). Linear artifacts reveal the SPOT 4 CCD distortions.

$R$ 3-D rotation matrix that accounts for the satellite roll, pitch, and yaw when $p$ was being acquired;

$T$ system reference change matrix from the orbital to the terrestrial coordinates system;

$\delta \quad$ correction brought on the orthorectification model by the GCPs to ensure precise coregistration of the orthorectified salve and the reference images;

$\lambda$ some positive scaling number such that the ray defined by $O(p)+\lambda \vec{u}(p)$ intersects the topography surface as defined by the DEM at $M$.

GCPs are automatically derived with high accuracy by optimizing $\delta$ on some designated pixels of the raw image, called image control points (ICPs), such that orthorectified patches centered on those ICPs have an average coregistration with the reference image as accurate as possible, as measured using subpixel correlation.

In practice, we do not distinguish between the instrument optical center and the satellite center of mass, and $\delta$ is a linear correction on each of the $X, Y$, and $Z$ component of the terrestrial look direction $T(p) R(p) \vec{u}_{1}(p)$. Although $\delta$ has the potential of correcting any defect from both the interior and exterior orientations, the linear correction mostly corrects for attitude drifts of the satellite. We then consider that $\delta$ corrects for any bias of the external orientation and that the remaining biases are only due to distortions from the interior orientation, i.e., the $\mathrm{CCD}$ distortions.

Given a pixel $p$, the direct orthorectification model determines its projection $M$ on the ground.

2) Calibration: Given the orthorectification model, the following procedure, introduced in Fig. 2, is used to compute the calibrated look direction $\vec{u}_{N}$ for all pixels in the slave image.
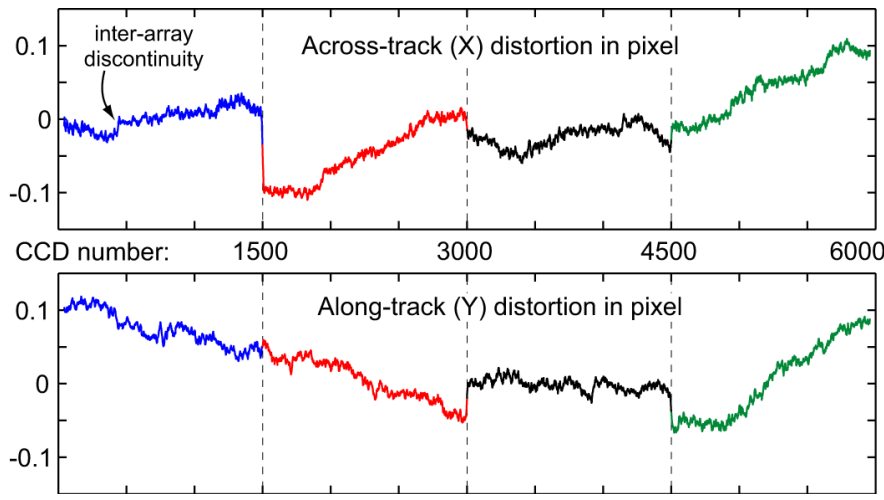

Fig. 5. Measured distortions, in pixels, in the focal plane of the SPOT 4-HRV1 panchromatic sensor. The CCD line sensor is composed of four CCD line arrays of 1500 pixels each (vertical dotted lines). Discontinuities on the edges of each array reveal their misalignment. Both across-track and along-track distortions are measured with an uncertainty below 0.01 pixel rms. Distortions of up to 0.12 pixel ( $~ 1.2 \mathrm{~m}$ on the ground) are estimated. All 6000 pixels are calibrated, except for the first 43 and the last 40 pixels due to border effect in the correlation analysis. The distortions for these missing pixels were later linearly extrapolated from the nearest 150 distortions to provide a complete calibration of the sensor.

1) Call $M$ the ground projection of the pixel $p$ by the direct model orthorectification. Orthorectify the raw slave image onto a $32 \times 32$ pixel patch $\mathbf{P}$ centered at $M$.

2) Compute the disparity $\vec{D}_{1}$ between $\mathbf{P}$ and the orthorectified reference image using subpixel correlation.

3) Find $M_{1}=M+\vec{D}_{1}$. Assign to $M_{1}$ its elevation $h_{1}$ according to the DEM.

4) Determine the new interior orientation look direction $\vec{u}_{N}$ such that $M_{1}(p)=O(p)+\lambda_{1}\left[T(p) R(p) \vec{u}_{N}(p)+\vec{\delta}(p)\right]$ for some $\lambda_{1}>0$ and under the constraint $\left\|\vec{u}_{N}(p)\right\|_{2}=1$. This yields

$$
\vec{u}_{N}(p)=R^{T}(p) T^{T}(p)\left(\frac{\overrightarrow{O M}_{1}}{\lambda_{1}}-\vec{\delta}(p)\right)
$$

with $\lambda_{1}$ determined from the constraint $\vec{u}_{N} \cdot \vec{u}_{N}=1$, which gives

$\lambda_{1}=\frac{\overrightarrow{O M}_{1} \cdot \vec{\delta}-\sqrt{\left(\overrightarrow{O M}_{1} \cdot \vec{\delta}\right)^{2}-\left(\|\vec{\delta}\|^{2}-1\right)\left\|\overrightarrow{O M}_{1}\right\|^{2}}}{\|\vec{\delta}\|^{2}-1}$.

We indeed have $\lambda_{1}>0$ since $\|\vec{\delta}\|^{2}<1$. Physically, this means that the correction on the orthorectification model has a smaller magnitude than the pointing vector to be corrected. This should always be true when the satellite is imaging its specified ground target.

5) Iterate for all pixels in the raw slave image that overlap with the reference image. See Appendix for details.

This procedure yields a calibration for each CCD element. It provides maximum redundancy because it is carried out for all lines in the raw image. Because pointing vectors (or look directions) are characteristic of the camera, they can be assumed constant over the image scanning time for a given CCD element, and they are "averaged" to give a more accurate calibration. For a given $\mathrm{CCD}$ element, averaging all the pointing 
vectors $\vec{u}_{N}^{i}$ means finding the unitary vector $\left\langle\vec{u}_{N}\right\rangle$ such that its direction is the mean direction of all unitary vectors $\vec{u}_{N}^{i}$. This is equivalent to finding $\left\langle\vec{u}_{N}\right\rangle$ that satisfies $\left\langle\vec{u}_{N}\right\rangle=$ $\arg \min _{u} \sum_{i}\left\|\vec{u}-\vec{u}_{N}^{i}\right\|^{2}$ for $\|\vec{u}\|=1$. Equivalently, the spherical coordinate angles $\left(\theta_{N}, \varphi_{N}\right)$ of $\left\langle\vec{u}_{N}\right\rangle$ are the minimizers of

$$
\begin{aligned}
f(\theta, \varphi)=\sum_{i}\left(1-\vec{u} \cdot u_{N}^{\vec{i}}\right), \\
\quad \text { for } \vec{u}=(\sin \varphi \cos \theta, \sin \varphi \sin \theta, \cos \varphi)^{T} .
\end{aligned}
$$

If we let $A, B$, and $C$ denote the coordinates of the vector $\sum_{i} \vec{u}_{N}^{i}$ in the spacecraft body fixed reference system, i.e., $A=$ $\sum_{i} \vec{u}_{N}^{i} \cdot \vec{X}, B=\sum_{i} \vec{u}_{N}^{i} \cdot \vec{Y}$, and $C=\sum_{i} \vec{u}_{N}^{i} \cdot \vec{Z}$, we find

$$
\begin{aligned}
\theta_{N} & =\arctan \left(\frac{B}{A}\right) \\
\varphi_{N} & =\arctan \left(\frac{A \cos \theta_{N}+B \sin \theta_{N}}{C}\right)
\end{aligned}
$$

by equating the partial derivatives of $f(\theta, \varphi)$ to 0 . For each CCD element, we can then determine a mean calibrated look direction $\left\langle\vec{u}_{N}\right\rangle$. In practice, to limit bias in the mean calibration, only calibration measurements resulting from a correlation with high signal-to-noise ratio and with ground disparities comprised within a physical range of a few meters are used. At this point, the interior orientation of the satellite is fully calibrated, and it is worth noting that no a priori knowledge on the camera parameters such as the focal length or the CCD sampling step in the focal plane have been used. The resulting calibration is therefore not biased even when these parameters are not known with enough accuracy.

We previously stated that the distortions of the optical system were primarily due to positioning errors of the CCD elements in the focal plane. Now that the camera interior orientation is calibrated, the focal plane distortions $\vec{d}$ (see Fig. 2) can be determined by looking at the difference between the projection of the calibrated and noncalibrated look directions $\left\langle\vec{u}_{N}\right\rangle$ and $\vec{u}_{1}$ in the focal plane. We have

$$
\vec{d}=\left(d_{x}, d_{y}, 0\right)^{T}=p^{\prime}-p=\frac{f}{r}\left[\frac{\left\langle\vec{u}_{N}\right\rangle}{\left|\left\langle u_{N}(z)\right\rangle\right|}-\frac{\vec{u}_{1}}{\left|u_{1}(z)\right|}\right]
$$

where $f$ is the instrument estimated focal length, $r$ is the sampling step of the CCD array, and $u(z)$ is the $Z$ component of $\vec{u}$, i.e., $u(z)=\vec{u} \cdot \vec{Z}$. For the SPOT 4 instrument, we used $r=$ $13 \mu \mathrm{m}$ [12]. The exact value of the focal length slightly varies depending on authors, and we followed the recommendation of [13] using $f=1084 \mathrm{~mm}$. Fig. 5 shows each component, e.g., across-track $d_{x}$ and along-track $d_{y}$, of the distortion $\vec{d}$ measured in the focal plane of the SPOT 4-HRV1 panchromatic sensor. Discontinuities are clearly seen on the edge of each CCD array at pixel multiples of 1500 . The uncertainty of this calibration model is better than 0.01 pixel rms and shows significant CCD distortions even within each single CCD array.

Noncalibrated look angles $\left(\Psi_{x}, \Psi_{y}\right)$, defined in Fig. 3, relate to the noncalibrated interior orientation look directions $\vec{u}_{1}$. In the same way, calibrated look angles $\left(\Psi_{x}^{N}, \Psi_{y}^{N}\right)$ can be derived from the calibrated interior look direction $\left\langle\vec{u}_{N}\right\rangle$. Fig. 6

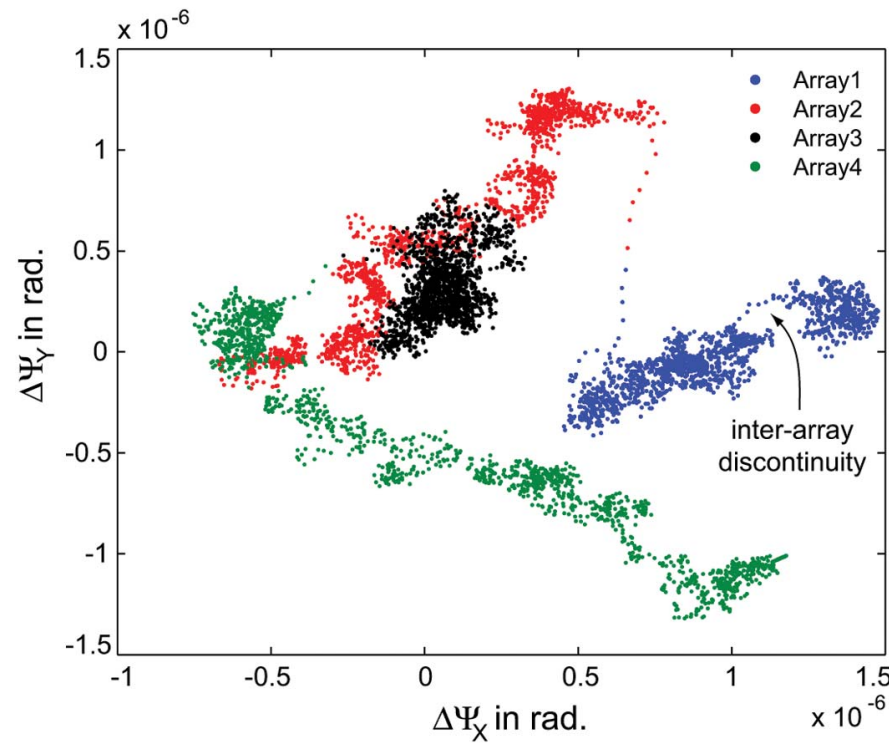

Fig. 6. Polar representation of the differences between calibrated and uncalibrated interior orientation look directions. We define $\Delta \Psi_{x}=\Psi_{x}^{N}-\Psi_{x}$, and $\Delta \Psi_{y}=\Psi_{y}^{N}-\Psi_{y}$, where $\left(\Psi_{x}, \Psi_{y}\right)$ and $\left(\Psi_{x}^{N}, \Psi_{y}^{N}\right)$ are derived from the noncalibrated $\left(\vec{u}_{1}\right)$ and calibrated $\left(\left\langle\vec{u}_{N}\right\rangle\right)$ interior orientation vectors. Each dot represents the look angle correction of a particular pixel. Colors are chosen to match those of Fig. 5. The clustering of the CCD arrays highlights the CCD array misalignment in the focal plane, and the linear trend of arrays 1,2, and 4 , shows that they are rotated in the focal plane. The CCD array 1 seems to be split into two clusters. The transition between these two clusters corresponds to the across-track discontinuity noticed in Fig. 5 around the pixel number 500 . The width of the dot cluster representing the CCD array 4 , about $0.1 \cdot 10^{-6} \mathrm{rad}$, is characteristic of the calibration uncertainty (not shown for clarity). At an altitude close to $830 \mathrm{~km}$, this calibration allows for a geometric accuracy that is about $8 \mathrm{~cm}$ on the ground, or 1/125 of the pixel size.

represents the difference between calibrated and noncalibrated look angles in the polar plane defined by $\left(\Delta \Psi_{x}, \Delta \Psi_{y}\right)$, with $\Delta \Psi_{x}=\Psi_{x}^{N}-\Psi_{x}$, and $\Delta \Psi_{y}=\Psi_{y}^{N}-\Psi_{y}$, for all pixels $p$ in the SPOT 4-HRV1 CCD sensor. This representation helps to visualize the kind of distortion that the CCD sensor suffers. On the first order, the clustering of each CCD array shows that discontinuities between arrays cause the worst defects. On a second order, the linearity in this polar plane of the points belonging to the CCD arrays 1,2 , and 4 shows internal rigid rotation of these arrays in the focal plane. On a third order, we can also point out interarray discontinuities, as seen on array 1 . It is also visible in Fig. 5 around the pixel number 500 in the across-track direction.

3) Spot Steering Mirror and Correction Model: The SPOT interior orientation look directions $\vec{u}_{1}$ account for the modeling of the steering mirror [20]. Therefore, the correction devised above cannot be directly applied to another image from the same sensor, acquired with a different incidence angle. We need to introduce the rotation matrix $R_{M}$ modeling the effect of the lateral steering mirror

$$
R_{M}=\left(\begin{array}{ccc}
\cos (\Theta) & 0 & -\sin (\Theta) \\
0 & 1 & 0 \\
\sin (\Theta) & 0 & \cos (\Theta)
\end{array}\right)
$$

where $\Theta$ is a rotation angle around the $Y$-axis (Fig. 3). From the SPOT ancillary data, we compute $\Theta=(s-48) \times 0.6^{\circ}$, where 


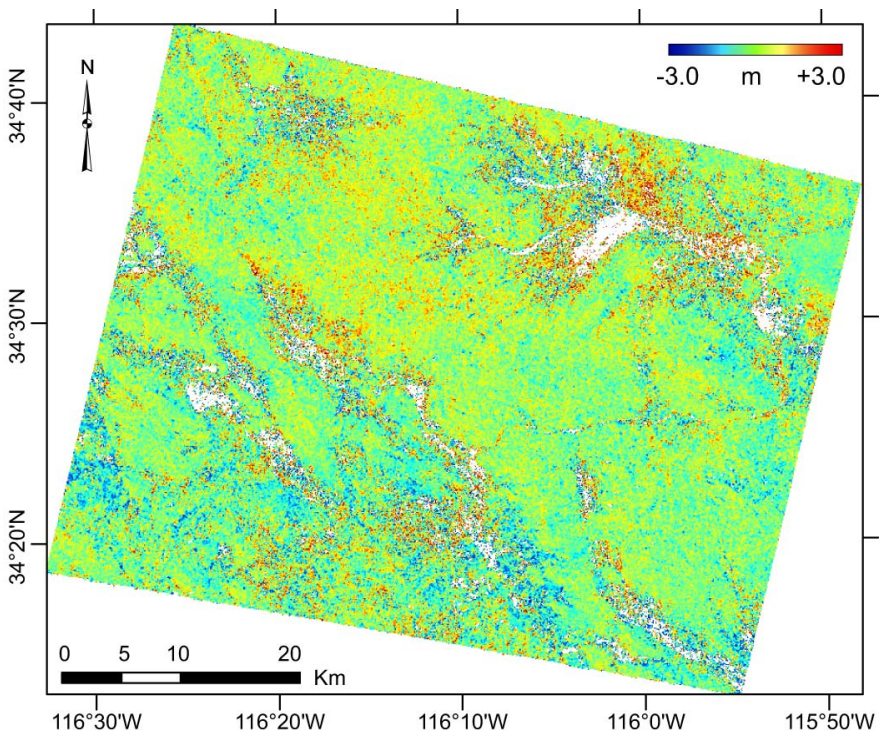

Fig. 7. Same experiment as in Fig. 4, but the derived CCD correction model has been accounted for during orthorectification. The absence of visible bias related to the CCD distortions validates the methodology.

$s$ is the step encoding the mirror rotation. We then define the correction model for the SPOT 1, 2, 3, and 4 satellites as

$$
\overrightarrow{d u} u_{0}(p)=R_{M}^{T}\left[\left\langle\vec{u}_{N}(p)\right\rangle-\vec{u}_{1}(p)\right]
$$

for all the 6000 pixels $p$ constituting the line sensor. In our particular example, the slave SPOT 4-HRV1 panchromatic image has a mirror step $s=46$; hence, $\Theta=-1.2^{\circ}$. This allows us to propose a general correction model for this particular sensor, assuming that the CCD distortions do not change over time. To apply this correction to another image from the same sensor, for example, to the image $I$, we correct the given interior orientation look directions $\vec{u}_{1}(p)_{I}$, for all pixels $p$, according to

$$
\vec{u}_{N}(p)_{I}=\vec{u}_{1}(p)_{I}+R_{M_{I}} \vec{d} u_{0}(p)
$$

where $R_{M_{I}}$ is the mirror rotation matrix associated with the image $I$, which is assumed constant for a given image. No images are indeed acquired when the mirror is rotating, and a safety lag time is set to allow the mirror position to rest until potential oscillations become negligible [21].

\section{RESUlTS}

Fig. 7 shows the correlation analysis between the SPOT 4 slave image when the interior orientation is corrected as described, and the SPOT 5 reference image. Compared with Fig. 4, it is clear that the CCD-induced distortions have been well corrected. This experiment validates the methodology presented and shows that the image from which the distortions are estimated is properly corrected. However, this experiment does not validate the way the mirror rotation is compensated. As the calibration model is used on the calibration image, the matrices $R_{M}$ and $R_{M_{I}}$ are identical, and they exactly compensate each other in the correction. Hence, this experiment is insensitive to mirror rotation uncertainties.

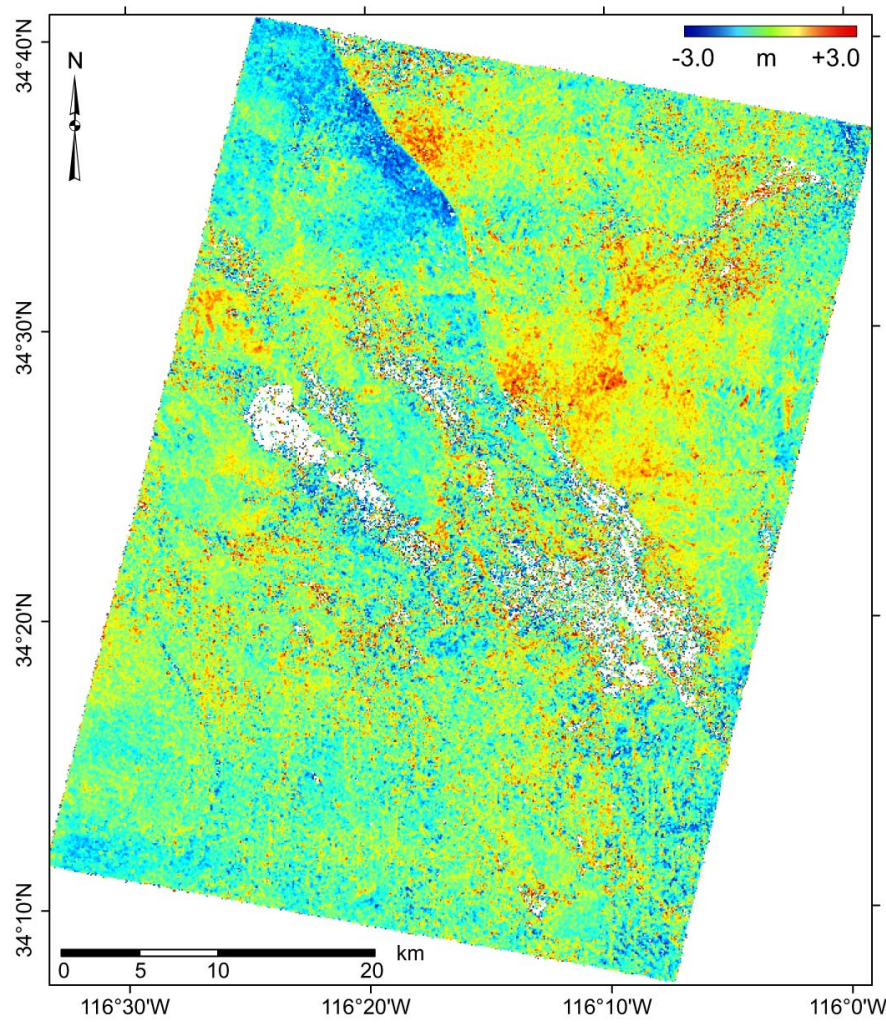

Fig. 8. Same experiment as in Fig. 1, but the derived CCD correction model for both SPOT 4 and SPOT 2 images have been accounted for during orthorectification. Although the SPOT 4 sensor was calibrated from an image acquired in 2000, it is applied here on a 1998 pre-earthquake image. The absence of CCD artifacts suggests that the CCD distortions of this particular sensor are stationary over the years. We also notice that the topography-induced artifacts have disappeared. The proposed methodology should then improve the quality of DEMs produced from stereoscopic image pairs acquired from the instruments considered in this paper.

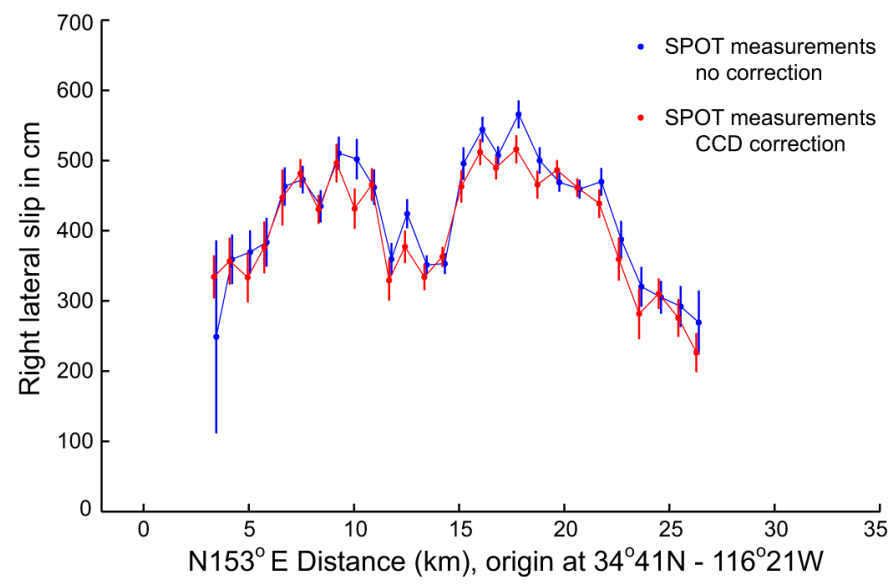

Fig. 9. Comparison of the right lateral slip along the fault trace estimated from the correlation analysis from Figs. 1 and 8. On this particular example, the CCD distortions induce up to $70-\mathrm{cm}$ bias on the measured fault slip. A right lateral slip is determined by projecting the horizontal slip vectors along the fault strike. Horizontal slip vectors are measured from linear least-square adjustment, on each side of the fault and on each NS and EW images, of stacked profiles perpendicularly running to the rupture. Profiles are stacked over a width of $880 \mathrm{~m}$ and a length of $8 \mathrm{~km}$.

Fig. 8 shows a more extended experiment and presents the same correlation analysis as in Fig. 1, but this time, the SPOT 4-HRV1 correction model that was estimated from the SPOT 
4-HRV1 image of 2000 is applied to the SPOT 4-HRV1 preearthquake image of 1998. To obtain a complete distortionfree image, the SPOT 2-HRV1 sensor was also calibrated using the same SPOT 5 reference image. No CCD artifacts remain; hence, we can conclude that the CCD distortions, at least between 1998 and 2000, can be considered stationary. This is an encouraging result suggesting that a single calibration of a particular instrument can hold for several years.

In seismotectonic studies, fault slip measurements are important in understanding the mechanics of seismic ruptures [3], [5], [7]. They can be determined from the correlation analysis of pre and post earthquake images by measuring the amplitude of discontinuities in profiles perpendicularly running through the fault. Thus far, it has been assumed that this procedure, which measures the relative displacement at the fault, is insensitive to artifacts from the imaging system. This is only true for artifacts whose wavelengths are much larger than the fault discontinuities, and this is unfortunately not the case of the CCD-induced distortions. In a particular setting where the fault is perpendicular to the satellite tracks, such measurements will not be biased, but if the CCD discontinuities are aligned with the fault offset, measurements will be inevitably corrupted. Fig. 9 compares the right lateral slip at fault from the offset field depicted in Figs. 1 and 8. The right lateral coseismic offset of the 1999 California Hector Mine earthquake is measured from the SPOT images before and after the CCD calibration is used. The discrepancy is up to $70 \mathrm{~cm}$ around kilometer 10 , where one of the CCD artifact crosses the fault near longitude $116^{\circ} 16^{\prime} \mathrm{W}$ (see Fig. 1). This shows that the incorrect account for CCD distortions can lead to significantly biased measurements of fault slip. Thus, by correcting topographic and registration biases, the CCD calibration allows better accuracy of measurements in change detection applications.

\section{Discussion AND CONCLUSION}

In this paper, we have presented a methodology for the inflight calibration of the interior model of pushbroom satellites. This calibration mostly aims at correcting the CCD distortions, which are the most common source of geometric artifacts encountered in these systems, and also any other stationary inaccuracies of the camera model. Interior orientation distortions are physically modeled as positioning errors on the location of the individual CCD elements and must be properly accounted for during orthorectification. This methodology requires the use of a reference image, ideally orthorectified. The topography of the calibration site should then be known with high accuracy. In particular, we have presented the calibration of the SPOT 4-HRV1 panchromatic sensor using a SPOT 5-HRG1 panchromatic image as calibration reference. The test site was in California, where the $1 / 3 \operatorname{arcsec}(\sim 9 \mathrm{~m})$ NED DEM is available.

The effectiveness of the calibration process relies on two main assumptions regarding the stationarity of CCD distortions. It is first assumed that the CCD distortions are constant during the short acquisition time of the calibration image (typically $<10 \mathrm{~s}$ ). Corrections determined at each line can then be averaged to provide a more precise calibration. In some peculiar cases, it could be argued that some mirror oscillations during the acquisition could perturb the correction model, but even if present, they in fact should be averaged to 0 while averaging the corrections over all lines (high-frequency oscillations [21]). This first assumption is thus very likely to be valid. The strength of a particular calibration is its ability to be applied to images other than the image it has been derived from. However, doing so is only valid if the CCD distortions are constant over much longer periods (typically a few years), which is the second assumption made here. In this paper, we successfully applied the correction derived from a 2000 image to a 1998 image. In addition, the same calibration model was applied to acquisitions from 2004 and 2006, investigated but not shown here [22]. These images were acquired at different latitudes, i.e., $12^{\circ} \mathrm{N}$ instead of $34^{\circ} \mathrm{N}$ for the California image used to derive the calibration, and at different seasons (summer versus winter), hence under different orbital conditions. In all these investigations, the residual distortions were at most 0.02 pixel. This residual is higher than the expected calibration accuracy detailed ( $\sim 0005-0.01$ pixel) but should be compared to the distortion errors when the calibration was not used, i.e., up to 0.12 pixel. However, the discrepancy observed between the expected and the measured calibration accuracy can be explained by several factors.

- As already discussed, the CCD distortions may not stay perfectly constant over long periods, and the calibration derived from the 2000 image may not be entirely valid from 1998 to 2006.

- The reference SPOT 5 image may exhibit some CCD distortions [23], and the NED DEM used for orthorectification is not ideal either. Hence, our orthoreference image certainly does not allow for a perfect calibration.

- Three years separate the SPOT 5 reference image from the SPOT 4 image from which the calibration is determined. Decorrelations related to land cover changes are visible in the correlation analysis of Fig. 4, and image-dependent biases may be present in the resulting calibration. For example, shadows on topographic features can bias the correlation analysis if the images are acquired at different seasons [1]. This could be minimized by averaging several calibrations derived from independent sets of images. Short acquisition periods between the reference and the calibration images should also be sought to minimize potential decorrelations.

- The mirror rotation of the calibration image is compensated for to derive a general calibration model, and then the mirror rotation of the image to be calibrated is accounted for to apply the correction. Mirror rotation angles are discretized every $0.6^{\circ}$, and these uncertainties add up to the final calibration inaccuracy. Again, averaging calibration models determined from sets of independent images should minimize the correction uncertainties.

Despite all these limitations, our results show that in-flight interior orientation calibration is beneficial, reducing internal camera biases by about one order of magnitude. Further work is still needed to better understand the cause and the time variability of the focal plane distortions, but we have shown that in the case of the SPOT 4-HRV1 panchromatic sensor, most 
of the CCD distortions could be thought as stationary errors for periods as long as eight years, even on different orbits. This fact suggests that distortions may mostly result from mechanical effects during the satellite launch that later remained, and that effects of on-orbit thermal variations on the satellite structure may only account for a more negligible part. Therefore, inflight interior orientation calibration is meaningful and should be generalized on all pushbroom systems designed to offer satisfying geometrical accuracy for, e.g., DEM generation and change detection applications.

The calibration models for the SPOT 2 and the SPOT 4-HRV1 panchromatic sensors described in this paper have been integrated to the free software package COSI-Corr, developed with Interactive Data Language and integrated in the Environment for Visualizing Images. This software is available from the Caltech Tectonics Observatory website (http://www. tectonics.caltech.edu/).

This paper validates that the CCD elements of optical sensors are subject to positioning errors on the order of $1 / 10$ of the pixel size. Images acquired by such sensors are therefore not exactly regularly sampled. Most orthorectification procedures rely on inverse orthorectification modeling, where a regular grid on the ground is back-projected in the satellite image plane. The orthoimage is produced by resampling the raw image, assumed regularly sampled, at the grid points back-projected in the image plane. This formulation is thought to produce rigorously built orthoimages while avoiding the problem of irregular resampling posed by the direct orthorectification modeling [1]. Because the raw images can no longer be assumed strictly regularly sampled, this paper suggests that a more explicit account of the irregular resampling problem, as treated in [24] for instance, might help to further improve the quality of orthorectified images.

\section{APPENDIX \\ DETERMINING THE OVERLAP BETWEEN THE SLAVE AND THE REFERENCE IMAGES}

To avoid unnecessary computations during calibration, it is crucial to determine beforehand the region in the raw slave image, which, once projected on the ground, will overlap with the orthorectified reference image. This can be automatically accomplished with the following algorithm.

1) Extract the ground coordinates (UTM) of the four corners of the orthorectified reference image. This defines a convex 4-polygon $P_{1}$.

2) Determine the ground coordinates (UTM) of the raw slave image corners using the direct orthorectification model. This defines a second 4-polygon $P_{2}$ in the orthorectified domain.

3) Compute the intersection of the interior of these two 4-polygons. This can be solved using SutherlandHodgman's polygon clipping algorithm [25]. The intersection is a new polygon $P_{3}$ in the orthorectified domain.

4) Map $P_{3}$ from the orthorectified domain to the geometry of the raw slave image. This is done by applying the inverse orthorectification model on each vertex of $P_{3}$.
5) The projection of $P_{3}$ in the raw slave image plane is assumed to be the polygon $P_{4}$, delimited by the inverse projection of the $P_{3}$ vertices. $P_{4}$ is then shrunk by the size of the correlation window that will be used during calibration to avoid edge effects, producing the polygon $P_{5}$. Distortions are then estimated for all pixels in the raw slave image that are contained within the $P_{5}$ polygon.

\section{ACKNOWLEDGMENT}

The authors would like to thank F. Ayoub (California Institute of Technology) who integrated the calibrations described in this paper to the COSI-Corr software, and I. Barisin (Oxford University) who applied the CCD calibration to the SPOT 4 images from 2004 and 2006, then allowing us to further test the stationarity assumption of the CCD distortions. Finally, this paper has benefited from excellent suggestions and critics from Renaud Binet (CEA, France) and an anonymous reviewer. This is Caltech Tectonics Observatory contribution \#81.

\section{REFERENCES}

[1] S. Leprince, S. Barbot, F. Ayoub, and J. P. Avouac, "Automatic and precise orthorectification, coregistration and subpixel correlation of satellite images, application to ground deformation measurements," IEEE Trans. Geosci. Remote Sens., vol. 45, no. 6, pp. 1529-1558, Jun. 2007.

[2] S. Leprince, F. Ayoub, Y. Klinger, and J. P. Avouac, "Co-registration of optically sensed images and correlation (COSI-Corr): An operational methodology for ground deformation measurements," in Proc. IGARSS, Barcelona, Spain, Jul. 2007, vol. 6, pp. 1943-1946.

[3] J. P. Avouac, F. Ayoub, S. Leprince, O. Konca, and D. Helmberger, "The 2005, $M_{w}$ 7.6 Kashmir earthquake, rupture kinematics from sub-pixel correlation of ASTER images and seismic waveforms analysis," Earth Planet. Sci. Lett., vol. 249, no. 3/4, pp. 514-528, Sep. 2006.

[4] N. Van Puymbroeck, R. Michel, R. Binet, J.-P. Avouac, and J. Taboury, "Measuring earthquakes from optical satellite images," Appl. Opt., vol. 39, no. 20, pp. 3486-3494, Jul. 2000.

[5] Y. Klinger, R. Michel, and R. King, "Evidence for a barrier model from Mw 7.8 kokoxili (Tibet) earthquake slip-distribution," Earth Planet. Sci. Lett., vol. 242, no. 3/4, pp. 354-364, Feb. 2006.

[6] R. Binet and L. Bollinger, "Horizontal coseismic deformation of the 2003 Bam (Iran) earthquake measured from SPOT-5 THR satellite imagery," Geophys. Res. Lett., vol. 32, no. 2, L02 307, 2005.

[7] S. Dominguez, J.-P. Avouac, and R. Michel, "Horizontal coseismic deformation of the 1999 Chi-Chi earthquake measured from SPOT satellite images: Implications for the seismic cycle along the western foothills of central Taiwan," J. Geophys. Res., vol. 108, no. B2, 2083, 2003.

[8] S. Leprince, E. Berthier, F. Ayoub, C. Delacourt, and J. P. Avouac, "Monitoring earth surface dynamics with optical imagery," EOS, Trans. Amer. Geophys. Union, vol. 89, no. 1, pp. 1-2, Jan. 2008.

[9] E. Berthier, H. Vadon, D. Baratoux, Y. Arnaud, C. Vincent, K. Feigl, F. Remy, and B. Legresy, "Surface motion of mountain glaciers derived from satellite optical imagery," Remote Sens. Environ., vol. 95, no. 1, pp. 14-28, Mar. 2005.

[10] M. Taylor, S. Leprince, J. P. Avouac, and K. Sieh, "Detecting coseismic displacements in glaciated regions: An example from the great November 2002 Denali earthquake using SPOT horizontal offsets," Earth Planet. Sci. Lett., 2007. to be published.

[11] S. Leprince, E. Berthier, F. Ayoub, C. Delacourt, and J. P. Avouac, "Monitoring Earth surface dynamics with optical imagery," in Proc. Amer. Geophys. Union-Fall Meeting, San Francisco, CA, Dec. 2007.

[12] SPOT User's Handbook, SPOT Image Corp., Reston, VA, 1990.

[13] T. Westin, "Inflight calibration of SPOT CCD detector geometry," Photogramm. Eng. Remote Sens., vol. 58, no. 9, pp. 1313-1319, Sep. 1992.

[14] T. Westin, "Interior orientation of SPOT imagery," in Proc. XVIIth ISPRS Congr., Commission I, Washington DC, 1992, vol. 29, pp. 193-198.

[15] E. Baltsavias, Z. Li, and H. Eisenbeiss, "DSM generation and interior orientation determination of IKONOS images using a testfield in Switzerland," in Proc. ISPRS Workshop High-Resolution Earth Imaging Geospatial Inf., Hannover, Germany, 2005. 
[16] K. Jacobsen, "Calibration of optical sensors," in Proc. ISPRS Commission I, Int. Calibration Orientation Workshop EuroCOW, Castelldefels, Spain, 2006.

[17] National elevation dataset, Reston, VA: U.S. Geol. Survey. [Online]. Available: http://ned.usgs.gov/

[18] R. Gachet, "SPOT5 in-flight commissioning: Inner orientation of HRG and HRS instruments," in Proc. XXth ISPRS Congr., Commission I, Istanbul, Turkey, 2004, vol. 35.

[19] Y. Teshima and A. Iwasaki, "Correction of attitude fluctuation of Terra spacecraft using ASTER/SWIR imagery with parallax observation," IEEE Trans. Geosci. Remote Sens., vol. 46, no. 1, pp. 222-227, Jan. 2008.

[20] S. Riazanoff, SPOT Satellite Geometry Handbook. Toulouse, France: SPOT Image, Jan. 2002

[21] E. Breton, A. Bouillon, R. Gachet, and F. Delussy, "Pre-flight and in-flight geometric calibration of SPOT5 HRG and HRS images," in Proc. ISPRS Commission I Symp., Pecora 15/Land Satellite Inf. IV Conf., Denver, CO, 2002.

[22] I. Barisin, S. Leprince, J. P. Avouac, B. Parsons, and T. Wright, "Deformation measurement for the September 2005 AFAR rifting event from subpixel correlation of spot images," in Proc. Amer. Geophys. Union-Fall Meeting, San Francisco, CA, Dec. 2007.

[23] A. Bouillon, E. Breton, F. D. Lussy, and R. Gachet, "SPOT5 HRG and HRS first in-flight geometry quality results," in Proc. SPIE-Sensors, Systems, Next-Generation Satellites VI, 2003, vol. 4881, pp. 212-223.

[24] A. Almansa, B. Rougé, and S. Jaffard, "Irregular sampling in satellite images and reconstruction algorithms," in Proc. 35th CANUM, Jun. 2003. [Online]. Available: http://www.fing.edu.uy/ almansa/HDR/ Almansa2003-CANUM-psample.pdf

[25] I. Sutherland and G. Hodgman, "Reentrant polygon clipping," Commun. $A C M$, vol. 17, no. 1, pp. 32-42, Jan. 1974.

Sébastien Leprince (S'06) received the Diplôme d'Ingénieur degree from the Ecole Supérieure d'Ingénieurs en Electronique et Electrotechnique, Paris, France, and the M.S. degree in electrical engineering in 2003 from the California Institute of Technology, Pasadena, where he is currently working toward the Ph.D. degree in electrical engineering.

His research focus was initially on high-frequency electronics and digital communication systems. His current research interests include image processing and analysis, optical acquisition systems, and remote sensing.
Pablo Musé received the Electrical Engineer degree from the Universidad de la República, Uruguay, Montevideo, in 1999, the Diplôme d'Etudes Approfondies degree in mathematics, vision and learning, and the Ph.D. degree in applied mathematics from the Ecole Normale Supérieure de Cachan, Cachan, France, in 2001 and 2004, respectively.

From 2005 to 2006 he was with Cognitech, Inc., Pasadena, CA, where he worked on computer vision and image processing applications. Since October 2006, he has been a Postdoctoral Scholar with the Division of Geological and Planetary Sciences, California Institute of Technology, Pasadena.

Jean-Philippe Avouac received the Diplôme d'Ingénieur degree from the Ecole Polytechnique, Palaiseau, France, in 1987, and the Ph.D. degree in geology from the Université Paris VII, Paris, France, in 1991

$\mathrm{He}$ is currently a Professor of geology with the Division of Geological and Planetary Sciences, California Institute of Technology, Pasadena. His research activity is focused on understanding deformation of the Earth's crust, with a special focus on mountain building processes. His group has contributed to methodological advances in a variety of techniques used to measure crustal deformation based on GPS, remote sensing (SAR and optical images), field geology, and geomorphology. 